\title{
Age-related observations of low mass pre-main and young main sequence stars
}

\author{
Lynne A. Hillenbrand \\ California Institute of Technology \\ MC 105-24, Pasadena, CA 91125 (USA) \\ email: lah@astro.caltech.edu
}

\begin{abstract}
This overview summarizes the age dating methods available for young sub-solar mass stars. Pre-main sequence age diagnostics include the Hertzsprung-Russell (HR) diagram, spectroscopic surface gravity indicators, and lithium depletion; asteroseismology is also showing recent promise. Near and beyond the zero-age main sequence, rotation period or vsin $i$ and activity (coronal and chromospheric) diagnostics along with lithium depletion serve as age proxies. Other authors in this volume present more detail in each of the aforementioned areas. Herein, I focus on pre-main sequence HR diagrams and address the questions: Do empirical young cluster isochrones match theoretical isochrones? Do isochrones predict stellar ages consistent with those derived via other independent techniques? Do the observed apparent luminosity spreads at constant effective temperature correspond to true age spreads? While definitive answers to these questions are not provided, some methods of progression are outlined.
\end{abstract}

Keywords. stars: pre-main-sequence, (stars:) Hertzsprung-Russell diagram (Galaxy:) open clusters and associations: general

\section{Techniques for assessing young star ages}

Standard stellar age dating techniques can be divided in to those which are purely empirical in nature and those which are more theoretically grounded. The former include measurements related to stellar kinematics and cluster membership, stellar rotation as derived from periodic photometric modulation or spectroscopic absorption line broadening, stellar chromospheric activity as measured by e.g. fractional $\mathrm{Ca}$ II $\mathrm{H} \& \mathrm{~K}$ or $\mathrm{H} \alpha$ line luminosity, stellar coronal activity as measured by soft x-rays, lithium depletion trends, and age-metallicity as well as age-velocity dispersion relations. Most of the empirical correlations have dependencies on the stellar mass in addition to the age effect which is sought, adding necessary complication to any analysis. The latter methods, those referenced more directly to theory, include location in the Hertzsprung-Russell (HR) diagram relative to calculated isochrones, theoretical nuclear burning as traced though e.g. lithium abundances, and Asteroseismological constraints.

Each of the above diagnostics is in principle also applicable in the pre-main sequence phase of stellar evolution, as well as to the zero-age and young main sequence phases. However, many of them are significantly diminished in value as quantitative pre-main sequence age indicators due to "saturation" or "degeneracy" effects. Specifically, the coronal and chromospheric activity indicators which generally decline in strength with advancing main sequence age (e.g. Mamajek \& Hillenbrand, 2008) are either very shallowly dependent on, or constant with, age for solar-type pre-main sequence stars. Rotational behavior likewise deviates from the monotonic spindown characteristic of main sequence angular momentum evolution, with significantly higher dispersion in rotation properties observed at ages younger than $\sim 200$ Myr. This is explained as remnant behavior related to earlier interaction of the stellar magnetosphere with the primordial circumstellar disk, 
specifically star-to-star variation in the time scale for star-disk coupling. Kinematics and cluster membership still apply as age diagnostics in the pre-main sequence phase, and are employed in the same "guilt by association" manner as utilized for main sequence clusters. However, the absolute age dating is more difficult.

Another method that is sometimes used as a relative age dating technique for the very youngest stars is the fractional infrared excess luminosity, or the shape of the midinfrared spectral energy distribution. While it is true that the vast majority of stars with remaining detectable primordial circumstellar dust are younger than $\sim 10 \mathrm{Myr}$, and that the vast majority of stars with so much dust that they are seen via scattered light or are still partially or totally self-embedded are younger than $\sim 1-2 \mathrm{Myr}$, there is no evidence for a monotonic relationship between circumstellar dust characteristics and absolute stellar age. On the contrary, there are strong arguments for significant dispersion in the amount of circumstellar dust (and gas) among stars aged less than $\sim 10 \mathrm{Myr}$ - even those located in the same cluster or association. Thus, while stellar youth is certainly indicated by the presence of circumstellar material, the quantitative use of circumstellar properties as stellar chronometers is not recommended and will not be discussed further here.

We are thus left with three stellar age dating methods that are both applicable and increasingly well-calibrated at young - pre-main sequence and zero-age main sequence ages: (1) the theoretical HR diagram or extinction-correction color-magnitude diagram, (2) inferences of $\log g$ vs. $\log \mathrm{T}_{\text {eff }}$ from spectra, and (3) lithium abundance measurements and depletion trends. There is also some promise from (4) asteroseismology but this method has not yet proved itself. In what follows I discuss each of these four techniques and recent results.

\section{Stellar age dating in regions of recent star formation}

Stars form within giant molecular clouds that become unstable to fragmentation and subsequent gravitational collapse to produce: stellar clusters, multiple star systems, and individual stars. The time for an individual protostar to collapse is related to the local sound speed, and is expected to be 0.1-0.2 Myr (Shu, et al. 1987). On larger spatial scales, two main theories of star formation suggest different regulating phenomena and therefore time scales for the start-to-finish process of star formation in a molecular cloud. Regulation by quasi-static ambipolar diffusion processes takes 3-10 Myr (Shu, 1977, Mouschovias, 1976) while regulation by turbulence dissipation occurs on the dynamical time scale of only 0.5-few Myr (Ballesteros-Paredes et al. 1999, Elmegreen 2000).

We can hope to probe the relative importance of these physical processes by studying the mean ages and detailed age distributions in regions of current and recent star formation. Our main tools are those mentioned above: stellar bolometric luminosities and the HR diagram, spectral diagnostics of stellar surface gravity, and measurements of Li I $6707 \AA$ abundances. The bulk of my discussion concerns HR diagrams.

\subsection{HR diagrams}

A good case study that informs our understanding of stellar ages and age spreads in star forming regions is the Orion Nebula Cluster (ONC). Hillenbrand (1997) published a synthesis of existing and new photometry and spectroscopy in this region, enabling the location of over 900 stars on the theoretical HR diagram. Now, new and better photometry from HST/ACS and ESO/WFI as well as over 600 new optical spectral types from WIYN/Hydra, Palomar/Norris, and Keck/LRIS are available. Also, recent estimates of the ONC distance place the cluster $\sim 15 \%$ closer than previously accepted values. Further, we now have a better understanding of the photometric variability trends and amplitude 
ranges of individual ONC stars, enabling use of median photometry. The improved data along with revisions in our understanding of the temperature and bolometric correction scales appropriate for young pre-main sequence stars makes it worth revisiting the finding of a substantial luminosity spread in the Hillenbrand (1997) study.

First attempts at revision are presented by Da Rio et al. (2009), considering very carefully the subtleties of young star de-reddening and the effects of accretion, and Reggiani et al. (poster at this meeting), considering only the least photometrically variable stars. These authors find essentially no reduction in the $\sim 1.5$ dex luminosity spread [or $\sigma\left(\log \mathrm{L} / \mathrm{L}_{\odot}\right) \approx 0.55$ dex at fixed $\left.\log \mathrm{T}_{e f f}\right]$ characteristic of the lower quality and single epoch Hillenbrand (1997) data. Thus, observational errors and biases, and known causes of scatter do not appear to be the main culprit in creating the large luminosity spreads that are observed in the ONC HR diagram.

Indeed, such apparent luminosity spreads have been seen for decades in young cluster and association HR diagrams. Literature from the 1960s and 1970s, e.g. the venerable Iben \& Talbot (1966) and Ezer \& Cameron (1967) showed them. Such early comparisons between data and theoretical pre-main sequence isochrones are primarily responsible for long standing paradigms such as "molecular clouds form stars for about 10 Myr" and "circumstellar disks last about 10 Myr." Although the above statements have been modified with better data and modern interpretation, the evidence for cluster luminosity spreads has persisted. Thus the questions remain: Are the apparent luminosity spreads real? Do they indicate true age spreads? Can we use them to infer star formation histories?

\subsubsection{HR diagram methodology}

Before embarking on these questions of HR diagram interpretation, it is important to review how stars are located in the HR diagram based on observational data and available techniques, as well as the accompanying complications to such procedures.

In practice, a spectral type determined at blue optical (BV), red optical (RI), or nearinfrared (YJHK) wavelengths is used along with a spectral-type- to-effective-temperature conversion to set the abscissa in the HR diagram. Photometry within some subset of optical or near-infrared bands is used along with the spectral type to calculate and correct for reddening, and then a bolometric correction appropriate to the spectral type is adapted in order to calculate the ordinate of bolometric luminosity.

Complications to this standard process that are unique to young stars include effects related to the ubiquitous presence of circumstellar disks for some portion of the early pre-main sequence (see Meyer, this volume). Accretion from the disk on to the star creates a hot excess which makes blue photometry "too blue," while thermal plus accretion emission from the inner disk makes red photometry "too red." Both phenomena confuse de-reddening procedures. The potential existence of both blue and red excess means that, in fact, there may be no truly photospheric wave band at which to apply bolometric corrections to the reddening corrected photometry. Furthermore, some young sources are not seen directly, but via light scattered through circumstellar disks or envelopes which leads to significant luminosity underestimation. For example, all Taurus scattered light sources sit on or below the zero-age main sequence. The extent to which scattered light affects other systems, in which it is not known from spatially resolved images, is unknown. Luminosity effects resulting from typical parameter distributions star plus disk systems were modeled by Kenyon \& Hartmann (1990) who found induced luminosity deviations relative to non-disked stars of $\sigma\left(\log \mathrm{L} / \mathrm{L}_{\odot}\right)<0.2$ dex. Other concerns for HR diagram construction at young ages include generally large values of visual extinction (>1-10 mag), uncertainty regarding the appropriate extinction law, and significant photometric variability at typically $<0.2$ mag levels though $>1$ mag in more extreme cases. 
Additionally present for the young stars are the usual complications affecting all HR diagram determinations. These include random errors due to spectral type and photometric uncertainties, and systematic errors deriving from unresolved multiplicity that result in luminosity overestimates (e.g. Simon et al. 1993).

\subsubsection{Checks on methodology}

Checks on our ability to locate young stars in the HR diagram are provided by binary and higher order multiple systems, whose components we expect to be coeval. Previous work in this area includes that by Hartigan et al. (1994), White et al. (1999) Prato et al. (2003), and Ammler et al. (2005). Recently, Kraus \& Hillenbrand (2009) have used more modern temperature and luminosity pairs based on improved photometry and spectral types from high spatial resolution data to determine that, indeed, the binaries and higher order multiples in the Taurus-Auriga region are more coeval than random pairings of member stars. However, while some multiple systems lie on theoretical isochrones (within the errors), others are significantly mismatched. It is unclear at present whether the observed effects can be attributed to random or systematic errors, or if they indicate true non-coevality, but the result should be kept in mind as we proceed to discuss luminosity spreads in clusters as a whole.

Totally independent checks on $\log \mathrm{L} / \mathrm{L}_{\odot}$ and $\log \mathrm{T}_{\text {eff }}$ conversions via the theoretical HR diagram to stellar masses come from comparison of such predictions to dynamical mass measurements. However, similarly independent checks of $\log \mathrm{L} / \mathrm{L}_{\odot}, \log \mathrm{T}_{\text {eff }}$ to stellar age predictions are more difficult to develop. At best, we can demand that the theoretical isochrones are parallel to observed cluster sequences, similar to the expectations for binaries and higher order multiples. We can also hope for consistency with other techniques, such as e.g. turn-off ages for the higher mass stars in the same cluster, surface gravity measurements, lithium abundance determinations, etc.

\subsubsection{HR diagram theory}

As we aim to use HR diagrams to gain knowledge about absolute stellar ages, in addition to investigating the evidence for or against spreads in age, it is important to discuss in brief the calibrating theory for the stellar age determinations.

As detailed in Hillenbrand et al. (2008), there are significant systematic effects between available theoretical predictions of pre-main sequence evolution. Specifically, the trend at sub-solar masses is that for a given location in the HR diagram, the youngest ages are those inferred from the D'Antona \& Mazzitelli (1994, 1997 and 1998 update) theory with increasingly older ages predicted by Yi et al. (2001, 2003, 2004), Swenson et al. (1993), Palla \& Stahler (1993, 1999), Siess et al. (2000), and finally the Baraffe et al. (1995, 1998) theory predicting the oldest ages. Age differences between these various track sets for the same $\log \mathrm{L} / \mathrm{L}_{\odot}, \log \mathrm{T}_{\text {eff }}$ pair rise to $\sim 0.75$ dex at the youngest ages!

Furthermore, with all isochrone sets, trends of stellar age with stellar mass are present, as widely reported in observational papers. Along any empirical isochrone $<50-80 \mathrm{Myr}$, the higher mass stars generally appear older than the lower mass stars. This suggests either that we are still missing physics associated with the initial appearance of stars in the HR diagram, or that the mass-dependent physics of stellar interiors is still not adequately understood. As detailed by Palla (this volume), additional complications to pre-main sequence evolutionary theory such as the effects of initial conditions (i.e. the "birthline"), disk accretion, stellar magnetic fields, and stellar rotation may explain some of the observed dispersion between observationally derived and theoretically predicted effective temperatures and luminosities. 


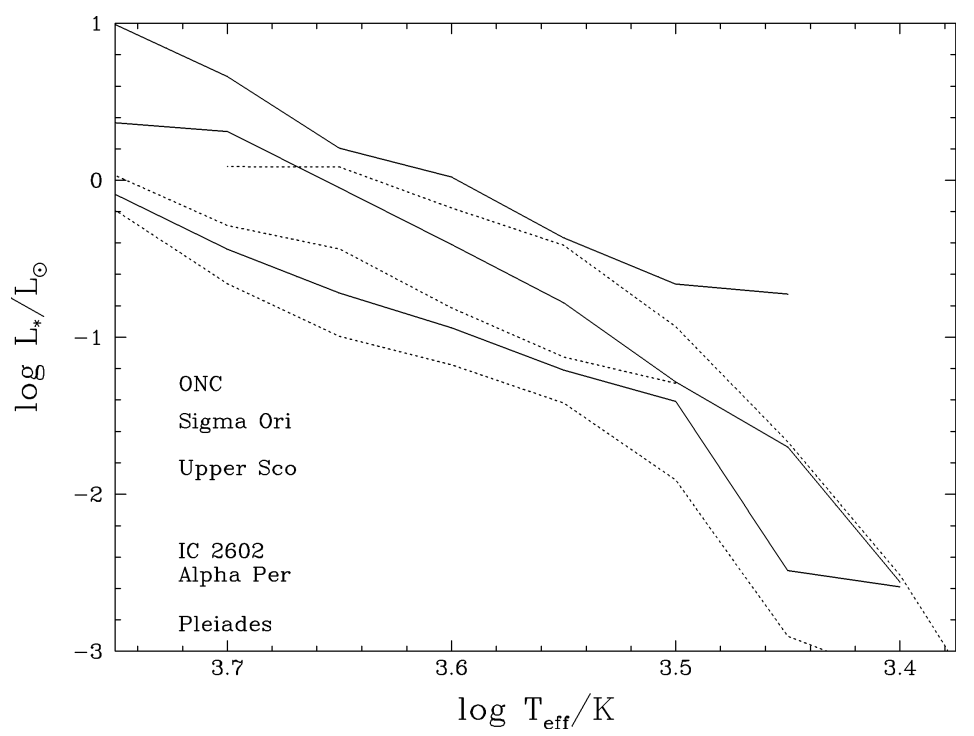

Figure 1. A sequence of empirical isochrones for representative young clusters and associations in the solar neighborhood. Shown is the median stellar bolometric luminosity as a function of stellar effective temperature for member star and brown dwarfs. Ordering in the legend corresponds to relative luminosity at $\log \mathrm{T}_{\text {eff }}=3.6$.

\subsubsection{Empirical results for young clusters}

I apply the methods outlined above and return now to the question of how well premain sequence clusters compare to theoretical isochrones. Shown in Hillenbrand et al. (2008) are the $\log \mathrm{L} / \mathrm{L}_{\odot}$ and $\log \mathrm{T}_{\text {eff }}$ distributions - represented as the mean and 1- $\sigma$ luminosity vs. binned effective temperature - for over 25 young clusters, associations, and currently active star forming regions within $500 \mathrm{pc}$ of the Sun. I take the mean luminosity with effective temperature sequence in each region as an empirical isochrone. As illustrated comparatively in Figure 1, there is good representation among this sample of stellar populations having ages from $<1$ Myr to just over 100 Myr. The median luminosities at fixed effective temperature span approximately $1.5 \mathrm{dex}$.

I quantify the luminosity spreads in each region by calculating the distribution of $\Delta \log \mathrm{L} / \mathrm{L}_{\odot}=\left[\log L_{\text {observed }}(i) / L_{\odot}-\log L_{\text {median }}\left(\log T_{\text {eff }}\right) / L_{\odot}\right]$, or the deviation of individual luminosities from the median value appropriate for the temperature. These distributions, also, are illustrated in Hillenbrand et al. (2008). In most cases, Gaussian fits appear to describe adequately the luminosity deviations, suggesting that random processes are the dominant contributor to the luminosity spreads.

In detail, the fitted dispersions to the $\Delta \log \mathrm{L} / \mathrm{L}_{\odot}$ distributions for somewhat older (>30 Myr) near-main sequence young clusters, such as the Pleiades, $\alpha$ Per, IC 2602, IC 2391 , and the Tucanae / Horologium Association, are low with $\sigma\left(\Delta \log \mathrm{L} / \mathrm{L}_{\odot}\right)=0.10$ 0.15 dex. We can take this as the typical luminosity dispersion that may be expected from the HR diagram placement methods described above. Towards younger ages, however, empirical dispersion increases substantially with $\sigma\left(\Delta \log \mathrm{L} / \mathrm{L}_{\odot}\right)=0.2-0.6$ dex for clusters younger than 3-10 Myr. These spreads may be compared to the $0.15-0.25$ dex dispersions estimated as plausible by Hartmann (2001) for young stellar populations in which significant accretion luminosity is present, and the even smaller spreads discussed by Burningham et al. (2005) as characteristic of young variables. For pre-main sequence 
contraction going roughly as $\mathrm{L} \propto \tau^{-2 / 3}$, the implied age dispersion from literal interpretation of observed luminosity dispersion is then $\sigma(\log \tau) \propto 1.5 \sigma(\log L)$.

However, it is not only the Gaussian width that is important to assess in considering cluster luminosity dispersion. Rather, it may be the subtle deviations from pure Gaussianity that convey the important information apropos, e.g. star formation history of a region, or other factors such as binary properties of the sample. Monte Carlo simulation of the luminosity distributions that accounts for these various details can help illuminate the important effects, and is discussed in a later section.

At this point I would like to (re-)emphasize that before any apparent luminosity dispersion is considered real, that observational fidelity must be verified so as to minimize any contaminating effects to the already complex interpretation of the luminosity spread phenomenon. First, we should ensure that we are considering only certain cluster or association members and regions that are not confused by superposed episodes of star formation. Next, we should strive to obtain exquisite photometry and high quality spectroscopy so as to reduce the influence of random observational errors. We should account for possible scattered light (causing luminosity underestimates) in young regions and multiplicity (causing luminosity overestimates) in all regions; although these both are systematic effects, they apply to only some portion of the population and therefore contribute to apparent luminosity spreads.

In summary, only pristine samples and the best data should be used in probing luminosity distributions. I turn now to discussion of potential correlates with $\Delta \log \mathrm{L} / \mathrm{L}_{\odot}$.

\subsubsection{Independent observational checks}

We can test the reality of the observed apparent luminosity spreads via their confirmation by independent observational means. Specifically, we can look for correlations between $\Delta \log \mathrm{L} / \mathrm{L}_{\odot}$ and surface gravity indicators or lithium abundance trends. Further, we can take advantage of the asteroseismological checks that have recently come to fruition for pre-main sequence stars in certain mass regimes. In addition to the discussion below, I refer the reader to clever techniques pioneered by Jeffries (this volume) and Naylor (this volume) which also provide checks on the observed apparent luminosity spreads in young clusters.

\subsection{Surface gravity diagnostics}

Low mass stars have a number of surface gravity sensitive spectral features in the red optical wavelength range - that most often used to classify such objects in modern studies - with others available at near-infrared wavelengths but not discussed here. For stars with spectral types later than $\sim \mathrm{M} 2$, the $\mathrm{CaH} 6975 \AA$ band and the Na I 8183,8195 $\AA$ doublet lines are surface gravity sensitive at ages younger than $\sim 20-30$ Myr (Schiavon et al. 1995, Slesnick et al. 2006). Towards later spectral types, beyond $\sim \mathrm{M} 6$ and extending well into the L types, the K I 7665,7699 doublet lines, and several VO bands are additionally useful surface gravity diagnostics at ages younger than $\sim 100$ Myr (Steele \& Jameson 1995, Kirkpatrick et al. 2008).

One test of the reality of the observed apparent luminosity spreads in young clusters is whether there is any correlation between $\Delta \log \mathrm{L} / \mathrm{L}_{\odot}$, the deviation from the median cluster luminosity normalized for effective temperature, and a quantitative index of surface gravity. With $L \propto R^{2} T_{e f f}^{4}$ and $g \propto M / R^{2}$, we expect for low mass stars of constant mass contracting along Hayashi (roughly constant temperature) tracks that $\log \mathrm{L} / \mathrm{L}_{\odot}$ and $\log g$ should be inversely correlated. Slesnick et al. (2008) demonstrate for stars in the Upper Sco region that objects with the same or similar measures of the surface gravity sensitive Na I $8190 \AA$ spectral index can have a broad range of luminosity. Although the 

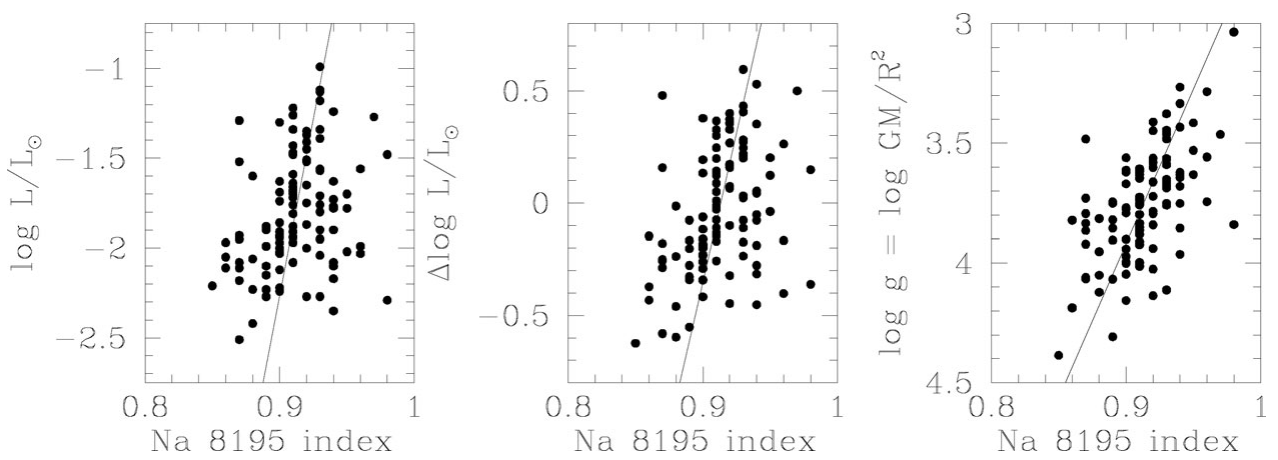

Figure 2. Based on data presented in Slesnick et al. (2008) for M4-M7 stars in the Upper Sco region. The left, middle, and right panels correlate $\log \mathrm{L} / \mathrm{L}_{\odot}$ (stellar bolometric luminosity), $\Delta \log \mathrm{L} / \mathrm{L}_{\odot}$ (deviation from mean luminosity normalized to effective temperature), and log $G M / R^{2}$ (surface gravity) computed from the pre-main sequence $\log \mathrm{L} / \mathrm{L} \odot$ and $\log \mathrm{T}_{\text {eff }}$ location in the HR diagram - all with the surface gravity sensitive Na I $8190 \AA$ spectroscopic index defined by Slesnick et al. Correlation coefficients and the linear least squares fits are poor for the left and middle panels, but -0.6 (inversely correlated) in the right panel with $0.27 \mathrm{dex} \mathrm{rms}$ for the displayed fit of $[\log g=(-12.9 \pm 0.7) \times N a I+(15.5 \pm 0.6)]$.

median age of the cluster is $\sim 5 \mathrm{Myr}$, individual stars with the same $\mathrm{Na}$ I index have ages predicted based on their luminosities from $<3$ to $>14$ Myr. If these luminosity-based ages are to be believed, we would expect corresponding differences in the $\mathrm{Na} I$ index.

What is observed is shown in Figure 2. Although there is significant scatter, it does appear that the surface gravities implied from HR diagram location do correlate in the expected way with a completely independent (spectroscopic) indicator of surface gravity. However, neither the straight $\log \mathrm{L} / \mathrm{L}_{\odot}$ nor the $\Delta \log \mathrm{L} / \mathrm{L}_{\odot}$ values exhibit similar correlation. Luminosity based ages for individual stars thus still warrant considerable skepticism, and cautions remain against uncritical assessment of observed apparent luminosity dispersion as true age dispersion. However, the quantitative results shown here do imply non-zero spread in both luminosity-based surface gravity and spectroscopic surface gravity, and thus by implication perhaps age at the several Myr level.

\subsection{Lithium $6707 \AA$ measurements}

Low mass stars burn both deuterium and lithium during the pre-main sequence evolutionary stages, essentially early steps in the hydrogen burning set of reactions that take place later on the main sequence. Contracting objects between $\sim 1-2.5 M_{\odot}$ undergo lithium burning processes for only a few tens of Myr to $<1 \mathrm{Myr}$, while those below $\sim 1 M_{\odot}$ and down to the hydrogen burning limit deplete their lithium essentially forever, and brown dwarfs burn only deuterium but never lithium (e.g. D'Antona \& Mazzitelli, 1994; Nelson et al. 1993). Lithium depletion trends in young pre-main sequence and main sequence populations have been used at sub-solar masses to estimate stellar ages, as discussed elsewhere in this volume. There is considerable scatter at constant age (e.g. within clusters) in both the observed equivalent widths and the derived abundances at constant mass or spectral type for stars younger than a few hundred Myr. Physically, this dispersion in surface abundance is likely related to the dispersion in rotation speeds over the same age range.

For any given star, lithium depletion is monotonically related to stellar age in the sense that lithium is never created via nuclear reactions, only destroyed. A test, therefore, of the reality of observed apparent luminosity spreads in young clusters is whether there 
is any correlation between $\Delta \log \mathrm{L} / \mathrm{L}_{\odot}$, the deviation from the median cluster luminosity normalized for effective temperature, and lithium abundance. Palla et al. $(2005,2007)$ have argued in the case of a small sample in the ONC that this is indeed the case, with isochronal and lithium depletion ages agreeing to within $5 \%$ in most cases based on the models of Siess et al. (2000). The agreement is particularly noted for those objects which sit low in the HR diagram relative to the main locus. However, it is just these stars which are suspected of being slightly foreground interlopers, part of the Orion Ic association which is indeed older and envelops the Orion Id (ONC) region. Thus, although intriguing and certainly an excellent way to test the conundrum of large luminosity spreads, in the particular case of the ONC there may be other complications which overshadow the main effect of this comparison.

An interesting case is that of St 34 in Taurus (White \& Hillenbrand, 2005), a nearequal mass binary with both components sitting low in the HR diagram relative to other Taurus members (isochronal age $\sim 8 \mathrm{Myr}$ ) and also near-fully lithium depleted (depletion age $>25 \mathrm{Myr}$ ). Otherwise, the star has all the characteristics typical of classical T Tauri stars: strong $\mathrm{H} \alpha$, He I, other accretion/outflow spectroscopic diagnostics, infrared excess, etc. It is thus either an unusually long-lived accretion disk system, or has had a somewhat unusual radial contraction and very unusual lithium depletion history. Another mysterious young object with apparent lithium depletion age much older than its isochronal age is Beta Pic group member HIP 112312 A (Song et al. 2002).

The existence of a few potentially anomalous objects like the examples above not withstanding, the correlation between lithium depletion and $\log \mathrm{L} / \mathrm{L}_{\odot}$ should be investigated more broadly. Recent studies of lithium in Taurus by Sestito et al. (2008) and in older nearby associations by Mentuch et al. (2008) provide some of the needed data.

\subsection{Asteroseismology}

Pulsational behavior in the Sun and other stars has offered important checks on our stellar interior models. Typically, e.g. in the case of the Sun as well as near the classical instability strip on the HR diagram, the pulsation mode is driven by opacity sources (the $\kappa$ mechanism). Some pre-main sequence stars of intermediate mass lie near this strip (Marconi \& Palla, 1998) and are being monitored for pulsations with a good number of detections to date (e.g. Zwintz et al. 2008 and references therein). Additionally, there is a prediction (Palla \& Baraffe, 2005) at the lowest masses that stellar/sub-stellar interior instability can be driven by deuterium burning ( $\epsilon$ mechanism) and also result in observable pulsational behavior. A narrow instability strip that is nearly parallel to the isochrones offers for these very low mass stars and brown dwarfs strong age constraints that are totally independent of the HR diagram - if pulsators can be found.

Many of the known brown dwarfs in star forming regions such as Chamaeleon I/II, Lupus, Ophiuchus, Upper Scorpius, IC 348, Sigma Ori, and the ONC lie near the predicted instability strip. Candidate objects are being monitored in thesis work by A.M. Cody (poster presented at this meeting) for photometric variability to determine which might be pulsators. Thus far, interesting variability at the right amplitudes $(<0.02 \mathrm{mag})$ and on the right time scales (several hours) has been detected through periodogram analysis.

Significant work of a very detailed nature still needs to be conducted on both the observational side and the theoretical side of pre-main sequence pulsations. In principle, however, asteroseismology is a powerful technique for assessing independently the stellar ages inferred from HR diagrams. 

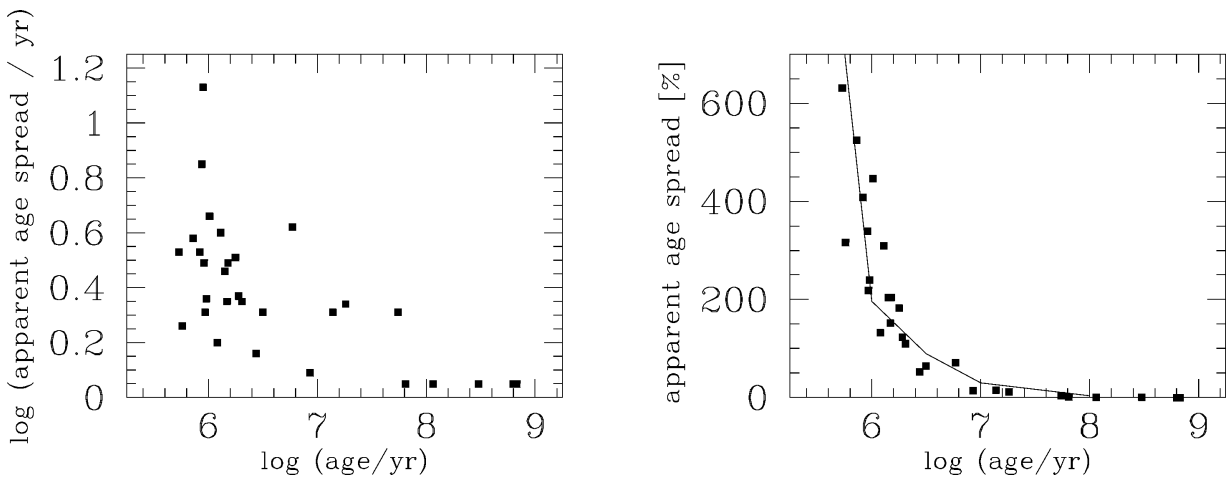

Figure 3. Left panel: 1-sigma log age dispersion versus mean log age as predicted from the $\log \mathrm{L} / \mathrm{L}_{\odot}$ and $\log \mathrm{T}_{\text {eff }}$ data by D'Antona \& Mazzitelli $(1997 / 1998)$. Other tracks generally yield older ages and even larger age dispersions. Right panel: corresponding percentage (linear) age error. The line indicates the expected age error introduced by an imposed luminosity scatter of 0.2 dex, and reproduces the observed "age scatter" for the youngest clusters though overpredicts the "age scatter" for older clusters where the actual luminosity spread is indeed lower. We can conclude that the observed luminosity dispersion is comparable to the luminosity spread expected from the random and systematic errors suffered during HR diagram placement, rather than being dominated by true age spread.

\section{Simple HR diagram simulations}

Having discussed young cluster HR diagrams and several independent checks on the observed apparent luminosity spreads, we turn briefly now to simulation of those luminosity spreads.

We consider as a first simplistic look, a situation in which the uncertainty $(\sigma)$ in individual values of $\log \mathrm{L} / \mathrm{L}_{\odot}$ which empirically characterizes the luminosity dispersion in older (main sequence) clusters, $\sim 0.1-0.2$ dex. We can then propagate such $\sigma(\log \mathrm{L})$ values into $\sigma(\log \tau)$ values, and compare to empirically inferred (from the observed $\Delta \log \mathrm{L} / \mathrm{L}_{\odot}$ distribution, or the apparent luminosity spread) values of $\Delta \log \tau$, or apparent age spread. I show in Figure 3 the predicted trend of $\sigma(\log \tau)$ vs $\log \tau$ compared to the trend actually inferred from young cluster HR diagrams. As can be seen, the assumption of luminosity errors typical of those on the main sequence leads to the expectation that age errors should rise towards younger ages to $>100-500 \%$ at ages $<1-3$ Myr.

Slesnick et al. (2008) performed a more realistic Monte Carlo simulation that projected on to the HR diagram the combined effects of various errors appropriate for late type premain sequence stars. An underlying $5 \mathrm{Myr}$ coeval population was masked by: photometric error of $0.025 \mathrm{mag}$ in each observation band (typical of observations), spectral type error of $1 / 2$ spectral subclasses, (typical of M-types), distance spread in the population (rendering the model cluster as deep as it is wide), and stellar multiplicity with $1 / 3$ of the systems being equal mass binaries (consistent with the mass ratios typically observed for low mass stars and brown dwarfs). The simulation resulted in a mean age and age dispersion totally consistent both visually and statistically with that found for the low mass population in the Upper Scorpius region. In other words, despite the apparent luminosity spread, no true age spread was needed in order to model the data.

Even more sophisticated Monte Carlo simulations were performed by A. Bauermeister in undergraduate thesis work. The models consider different possibilities for both evolutionary tracks and star formation histories, along with realistic input error and binary distributions in order to simulate cluster age spreads. The simulations can be analyzed 
in the same way as empirical data, e.g. calculating for the resulting HR diagrams the median luminosity as a function of effective temperature, the dispersion and the detailed shape of the $\Delta \log \mathrm{L} / \mathrm{L}_{\odot}$ distribution, and the slope of $\log \mathrm{L} / \mathrm{L}_{\odot}$ vs $\log \mathrm{T}_{\text {eff }}$ fit over a limited spectral type range.

Results thus far (as reported previously in Hillenbrand et al. 2008) indicate that the main effects of random errors are on the widths of the Gaussian core in the $\Delta \log \mathrm{L} / \mathrm{L}_{\odot}$ distributions, and the main effects of binaries are on the shape of the high side of the $\Delta \log \mathrm{L} / \mathrm{L}_{\odot}$ distribution. True age spread, if present, may be detectable as additional spread in $\Delta \log \mathrm{L} / \mathrm{L}_{\odot}$ present on both the high and low luminosity sides. We have found from extensive K-S testing that when observational errors are modest $(\sim 10 \%)$ and binarity properties of the underlying population are well understood, age spreads larger than $\sim 15-20 \%$ can be distinguished from no age spread or a "burst" star formation scenario. We continue to test the various parameter spaces, including variation of the star formation history (e.g. burst, constant, gaussian, linearly/exponentially increasing or decreasing). We also find that the fitted slope in $\log \mathrm{L} / \mathrm{L}_{\odot} \mathrm{vs} \log \mathrm{T}_{\text {eff }}$ can inform the choice of evolutionary tracks, modulo the binarity properties.

\section{Findings and implications}

Pre-main sequence evolutionary tracks: (1) vary significantly and systematically between theory groups; (2) under-predict stellar masses by $30-50 \%$; (3) under-predict likely low-mass stellar ages by $30-100 \%$; and (4) over-predict likely high-mass stellar ages by $20-100 \%$. The above imply large and systematic uncertainties in both mass and age distributions for young low mass populations, and hence: star formation histories in molecular clouds, disk evolutionary time scales, and angular momentum evolutionary time scales.

The reality of the observed apparent luminosity spreads in recently star forming regions can be tested via detailed correlation of the $\Delta \log \mathrm{L} / \mathrm{L}_{\odot}$ distributions with surface gravity indicators, lithium abundance measurements, and perhaps soon seismology checks in certain mass regimes. HR diagram simulations that account for plausible error, binary and other astrophysical effects are needed in order to determine the relevant luminosity spread or $\sigma(L)=\sqrt{\sigma_{\text {observed }}^{2}(L)-\sigma_{\text {understood }}^{2}(L)}$ that might then be assessed as a real luminosity spread for inference of any extended star formation history.

At present, there is only marginal or no strong evidence for moderate age spreads in young clusters. However, this conclusion does not preclude the "popcorn" effect for cluster star formation history, in which a few stars form first, preceding the main event, and a few stars lag, forming last - just like an episode of popcorn production relative to the interval between popcorn events in a typical household microwave oven or other popcorn nursery.

\section{Acknowledgement}

This presentation has included results established by my collaborators on various projects: Massimo Robberto and the HST Orion Treasury team plus Aaron Hoffer on the ONC, Adam Kraus on multiplicity and Taurus scattered light sources, Catherine Slesnick plus Davy Kirkpatrick on young star surface gravities, Russel White plus Amber Bauermeister on HR diagram simulations, and Ann Marie Cody on brown dwarf pulsations.

\section{References}

Ammler, M., Joergens, V., \& Neuhauser, R., 2005, A\&A, 440, 1127 
Ballesteros-Paredes, J., Hartmann, L., \& Vázquez-Semadeni, E., 1999, ApJ, 527, 285

Baraffe, I., Chabrier, G., Allard, F., \& Hauschildt, P. H. 1995, ApJL, 446, 35

Baraffe, I., Chabrier, G., Allard, F., \& Hauschildt, P. H. 1998, AA, 337, 403

Burningham, B., Naylor, T., Littlefair, S. P., \& Jeffries, R. D., 2005, MNRAS, 363, 1389

D'Antona, F. \& Mazzitelli, I. 1994, ApJS, 90, 467

D'Antona, F. \& Mazzitelli, I. 1997, in Cool stars in Clusters and Associations, ed. R. Pallavicini, \& G. Micela, Mem. S. A. It., 68, 807

Da Rio, N., Robberto, M., Soderblom, D. R., Panagia, N., Hillenbrand, L. A., Palla, F., \& Stassun, K., 2009, ApJ, submitted

Elmegreen, B. G., 2000, ApJ, 530, 277

Ezer, D. \& Cameron, A. G. W., 1967, Can. J. Phys., 45, 3429

Hartigan, P., Strom, K. M., \& Strom, S. E., 1994, ApJ, 427, 961

Hartmann, L. W., 2001, AJ, 121, 1030

Hillenbrand, L. A., 1997, AJ, 113, 1733

Hillenbrand, L. A., Bauermeister, A., \& White, R. J., 2008, ASPC, 384, 200

Iben, I. \& Talbot, R., 1966, ApJ, 144, 968

Kenyon, S. J. \& Hartmann, L. W., 1990, ApJ, 349, 197

Kirkpatrick, J. D., Cruz, K. L., Barman, T. S., Burgasser, A. J., et al., 2008, ApJ, 689, 1295

Kraus, A. L. \& Hillenbrand, L. A., 2009, ApJ, submitted.

Mamajek, E. E. \& Hillenbrand, L. A., 2008, ApJ, 687, 1264

Marconi, M. \& Palla, F., 1998, ApJ, 507, 141

Mentuch, E., Brandeker, A., van Kerkwijk, M. H., Jayawardhana, R., \& Hauschildt, P. H., 2008, ApJ, 689, 1127

Mouschovias, T. C. 1976, ApJ, 207, 141

Nelson, L. A., Rappaport, S., \& Chiang, E., 1993, ApJ, 413, 364

Palla, F. \& Barraffe, I., 2005

Palla, F., \& Stahler, S. W. 1993, ApJ, 418, 414

Palla, F., \& Stahler, S. W. 1999, ApJ, 525, 772

Palla, F., Randich, S., Pavlenko, Y. V., Flaccomio, E., \& Pallavicini, R., 2007, ApJ, 659, L41

Palla, F., Randich, S., Flaccomio, E., \& Pallavicini, R., 2005, ApJ, 626, L49

Prato, L., Greene, T. P., \& Simon, M., 2003, ApJ, 584, 853

Schiavon, R. P., Batalha, C., \& Barbuy, B., 1995, A\&A 301, 840

Sestito, P., Palla, F., \& Randich, S, 2008, A\&\&A, 487, 965.

Shu, F. H., 1977, ApJ, 214, 488

Shu, F. H., Adams, F. C., \& Lizano, S. 1987, ARAA, 25, 23

Siess, L., Dufour, E., \& Forestini, M. 2000, A\&A, 358, 593

Simon, M., Ghez, A. M., Leinert, Ch., 1993, ApJ, 408, L33

Song, I., Bessell, M. S., \& Zuckerman, B., 2002, ApJ, 581, L43

Slesnick, C. L., 2008, PhD thesis, California Institute of Technology

Slesnick, C. L., Carpenter, J. M., \& Hillenbrand, L. A., 2006, AJ, 131, 3016

Slesnick, C. L., Hillenbrand, L. A., \& Carpenter, J. M., 2008, ApJ, 688, 377

Steele, I. A. \& Jameson, R. F., 1995, MNRAS, 272, 630

Swenson, F. J., Faulkner, J., Rogers, F. J., \& Iglesias 1994, ApJ, 425, 286

White, R. J. \& Hillenbrand, L. A., 2005, ApJ, 621, L65

White, R. J., Ghez, A. M., Reid, I. N., \& Schultz, G., 1999, ApJ, 520, 811

Yi, S., Kim, Y.-C., \& Demarque, P. 2003, ApJS, 144, 259

Yi, S., Demarque, P., \& Kim, Y.-C., 2004, Ap\&SSS, 291, 261

Yi, S., Demarque, P., Kim, Y. -C., Lee, Y.-W., Ree, C. H., Lejeune, T., \& Barnes, S. 2001, ApJS, 136, 417

Zwintz, K., 2008, ApJ, 673, 1088

\section{Discussion}

R. Mathieu: This is the session where we need to address a very important topic for this symposium: What is the meaning of $t=0$ ? (Follow up comment: This issue underlies any 
attempt to define or test coevality in very young binaries or associations. Reversing the question, a coeval ONC population may constrain formation processes and correlations of formation across clouds?)

L. Hillenbrand: Hayashi would say $t=0$ corresponds to when the boundary is crossed establishing hydrostatic equilibrium. However, a further concept is that of the stellar birthline or the maximum $R(M)$ that establishes the $L$ and $T$ at which the star first "appears" in the HRD and begins its contraction vertically downward. Now, even if we agree on this, a further problem is the interaction of a young pre-main sequence star with its disk. These stars are accreting so they are increasing in mass by maybe $10 \%$ over the pre-MS phase, which causes motion in the HRD towards higher $T$ and $L$. Further, the clock can be "re-set" by outburst events that increase the luminosity significantly; several of these may occur in the first Myr or more, perhaps contributing to the luminosity dispersion in clusters.

F. WALTER: You have done an extraordinary job compressing a very broad topic into a short period. Consequently, you had to gloss over many details. Two aspects of the environment: 1. The Orion Nebula is viewed through the Orion OBIc association - OBIc is older and surely contaminates the sample to some extent. 2 . There may be a difference in age spreads between $\mathrm{OB}$ associations and $\mathrm{T}$ associations. In the former, feedback from the massive stars may terminate low mass SF by dispersing the gas and dust; no such mechanism exists in the $\mathrm{T}$ associations.

L. Hillenbrand: 1. I very much agree about the foreground contamination from the Orion Ic population which veils the Orion Id population I discussed. Some of these stars are likely present as the low luminosity outliers in the HRD. 2. One of Francesco Palla's slides I did not show included an argument based on the multiplicity of the massive ONC trapezium stars. Francesco demonstrated the locations of these individual components in the HRD and wanted us to appreciate that they are right on the birthline. Therefore, he says, the massive stars indeed formed last and may have terminated star formation as you say.

B. Weaver: Answer to the question of where is $t=0$ ? Choose a unique time which is independent of idiosyncratic starting conditions; therefore: use the ZAMS and use negative time for the PMS For Francesco: why not run models backwards in time to handle starting condition problems?

L. HillenBRAND: Other than reminding us of the meaning of "quasistatic contraction" I think I should defer this to a theorist (see Pinsonneault comment \#1).

M. Pinsonneault: 1) Comment: One would expect stars to converge to a unique state on a thermal timescale, so the range in true ages should be comparable to the range in assembly timescales. One does have difficulty in interpreting stars at the birthline.

2) Question: We know from ZAMS eclipsing binaries that spots can affect stellar radii. What information do we have about temperature variations in protostars?

L. Hillenbrand: 1. See Bruce Weaver's comment above. 2. Surely the stars are spotted, both in the traditional sense of cool spots related to the rotation-driven chromospheric and coronal activity, and because of hot spots due to accretion within rings at high latitudes and having filling factors of a few to maybe $10 \%$. The temperature inferred from low-resolution spectra of pre-MS stars do not account for any such effects. 
E. Jensen: You mentioned cleaning up the Orion Nebula cluster analysis by removing photometrically variable stars. While this will improve the HR diagram, will it bias you toward or against particular types of stars?

L. Hillenbrand: I don't see any evidence that the median luminosities or effective temperatures are biased, although the dispersion in luminosity is reduced. However, if it is true that so-called CTTS sit higher in the HRD than WTTS as Palla and Bouvier argue for Taurus and if the large-amplitude variable sample is dominated by CTTS as we would expect from accretion effects, your concern is valid.

I. KING: I understand that your beautiful CMD of Orion came from a mosaic of considerable size. What are the chances of getting a second epoch of observation, to get proper motions and cleanup the CMD?

L. HillenBRAnd: There is both archival data and new observations planned with HST over smaller portions of the area covered in the Robberto et al. program that are geared towards astrometric studies of the ONC. I recall the beautiful demonstration of the power of such techniques by you and Jay Anderson in NGC 6397.

M. Meyer: Comment: If one constrains an age spread to be coeval it seems to me that would constrain the distribution of infall rates (defining a plausible range of birthlines, cf. Hartmann et al. 1997. Question: In addition to the upper limits to the age spreads in OB associations and Taurus have you constrained them to be below some age in other low-mass $\mathrm{T}$ associations?

L. Hillenbrand: 1. Yes, in addition to all of the other effects already mentioned, any remaining luminosity spread will reflect some combination of spread in initial conditions (one of Palla's main points) plus any intrinsic age spread. 2. I do not yet have a rigorous analysis of each of the regions I discussed observationally. However, what you suggest is exactly the goal of this work.

R. Mathieu: We have been working on this issue for decades, and the issue of coeval formation remains uncertain. Is there a "killer" observation (or observations) that would/will resolve this?

L. Hillenbrand: I think membership constraints from kinematics are most crucial, so Gaia and other astrometric studies will be very important, as will large radial velocity surveys. 


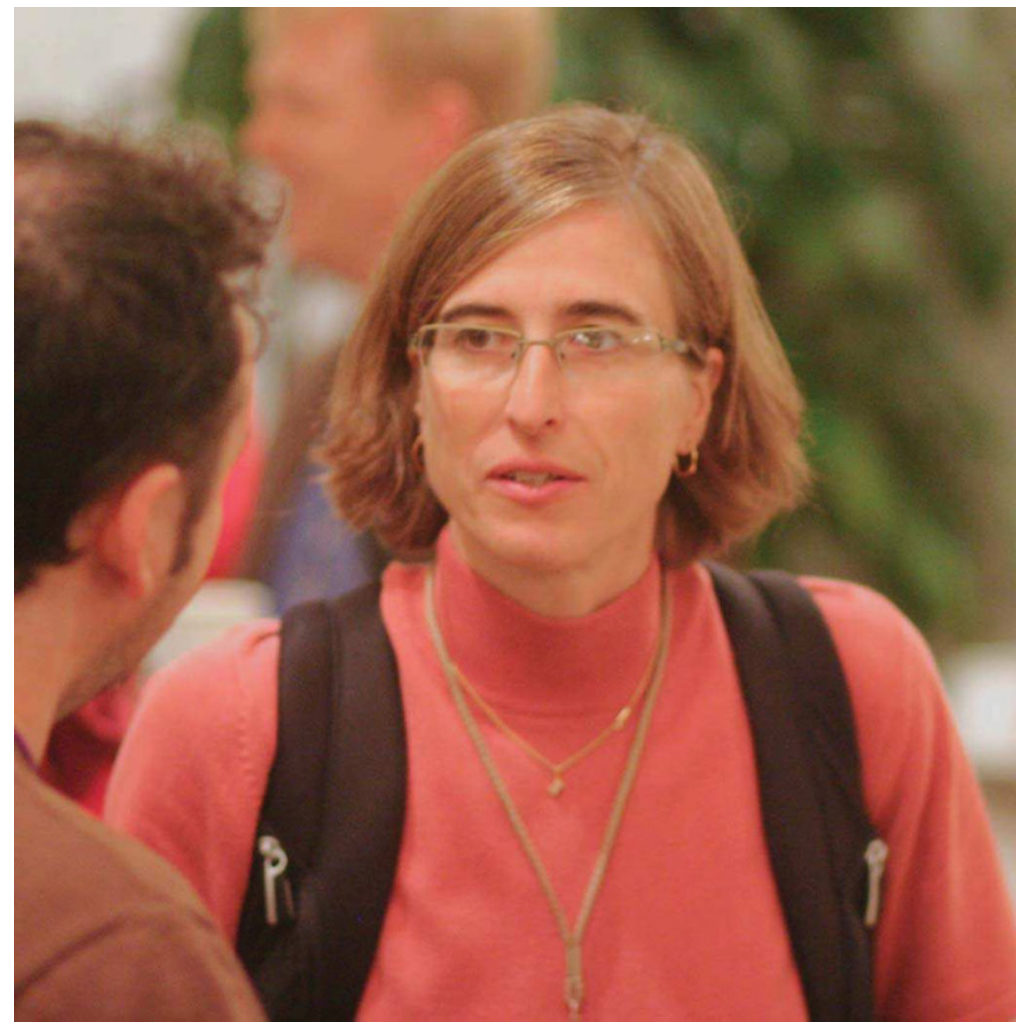

Lynne Hillenbrand

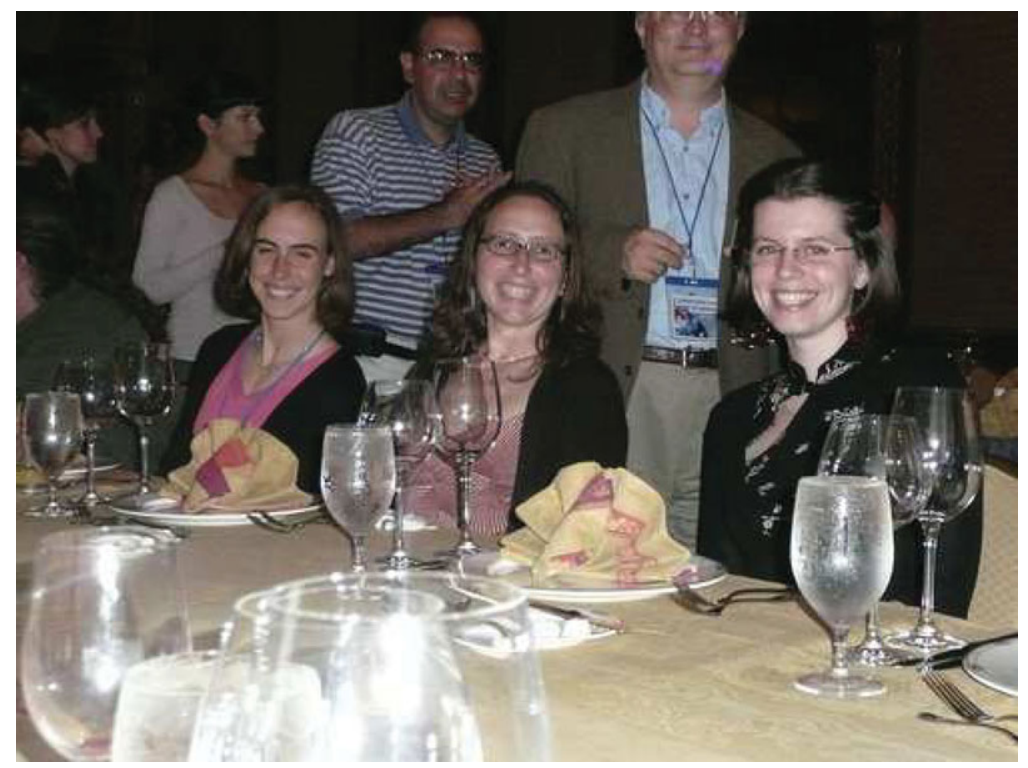

Ann Marie Cody, Kelle Cruz, and Anna Frebel 